\title{
Avaliação do tempo de evacuação de salas de aula com diferentes soluções de projeto ${ }^{1}$
}

\author{
Assessment of the evacuation time of classrooms \\ with different project solutions
}

\author{
Manuela Marques Lalane Nappi ${ }^{1}$ \\ Ivana Righetto Moser ${ }^{2}$ \\ João Carlos Souza ${ }^{3}$
}

\footnotetext{
1 Doutora em Arquitetura e Urbanismo pela Universidade Federal de Santa Catarina, Mestre em Engenharia Civil pela Universidade Federal de Santa Catarina e Graduada em Arquitetura e Urbanismo pela Universidade Federal de Santa Catarina. Pesquisadora no Programa de Pós-Graduação em Arquitetura e Urbanismo da Universidade Federal de Santa Catarina, Brasil.

E-mail:lalppi.ms@gmail.com

http://lattes.cnpq.br/7566130116889159 (D) http://orcid.org/0000-0001-7636-8539

2 Mestre em Arquitetura e Urbanismo pela Universidade Federal de Santa Catarina e graduada em Engenharia Civil pela Universidade Federal de Santa Catarina, Brasil.

E-mail:irighetto@yahoo.com.br

http://lattes.cnpq.br/5798549131320234 (1) http://orcid.org/0000-0003-3759-8811

3 Doutor em Engenharia de Produção pela Universidade Federal de Santa Catarina, Mestre em Engenharia de Produção pela Universidade Federal de Santa Catarina e graduado em Engenharia Civil pela Universidade Federal de Santa Catarina. Professor titular da Universidade Federal de Santa Catarina, Brasil.

E-mail:joao.carlos@ufsc.br $\underline{\text { http://lattes.cnpq.br/7323531303467032 }}$ http://orcid.org/0000-0002-4849-5810
}

\begin{abstract}
RESUMO: Sabe-se que o comportamento de pedestres em situações de emergência apresenta grande influência no tempo total de evacuação, tornando-o foco de muitas pesquisas no campo da física. Por outro lado, experimentos que reproduzam situações de pânico com seres humanos mostram-se impraticáveis devido a preocupações éticas e de segurança. Tem-se como objetivo, portanto, a simulação computacional de cinco diferentes cenários para uma sala de aula de 32 alunos, a fim de comparar os fluxos de pedestres quando da existência de uma ou duas saídas, além de duas disposições diferentes de mobiliário. Analisa-se ainda a correspondência dos resultados com uma situação real de evacuação registrada por câmera de vídeo e orientada pelo professor, descrita em Yang, Wu e Li (2011). As simulações foram realizadas em um software baseado em Forças Sociais e os resultados apontam a efetividade dos cenários com duas saídas, bem como a relevância do treinamento dos usuários.

Palavras-chave: Arquitetura. Evacuação emergencial. Simulação de fluxos. Soluções projetuais. Treinamento de usuários.
\end{abstract}

\begin{abstract}
It is known that the behavior of pedestrians in emergency situations has a great influence on the total evacuation time, making it the focus of much research in the field of physics. On the other hand, experiments that reproduce situations of panic with humans are impractical due to ethical and safety concerns. Therefore, the purpose of this research is the computational simulation of five different scenarios for a classroom of 32 students, in order to compare pedestrian flows when one or two exits exist, in addition to two different furniture arrangements. It is also analyzed the correspondence of the results with a real situation of evacuation registered by video camera and guided by the teacher, described in Yang, Wu e Li (2011). The simulations were carried out in software based on Social Forces and the results point to the effectiveness of the scenarios with two outputs, as well as the relevance of users' training.

Keywords: Architecture. Emergency evacuation. Simulation of flows. Design solutions. Users' training.
\end{abstract}

${ }^{1}$ O presente trabalho foi realizado com apoio da Coordenação de Aperfeiçoamento de Pessoal de Nível Superior Brasil (CAPES) - Código de Financiamento 001. 


\section{INTRODUÇÃo}

Para Helbing et al. (2005) projetar instalações para pedestres representa uma arte que exige fluxos eficientes. A pesquisa sobre comportamento de pedestres e multidões é altamente multidisciplinar. Ela envolve atividades de cientistas, psicólogos, sociólogos, biólogos, físicos, cientistas da computação, etc. O que explica a existência de opiniões controversas sobre o assunto como, por exemplo, em relação ao conceito de pânico, à explicação de padrões de movimento coletivos, ao melhor conceito de modelagem, entre outros (HELBING; JOHANSSON, 2011).

Uma vez que é considerado impraticável criar condições experimentais com humanos, pesquisas neste campo representam um grande desafio (SHIWAKOTI; SARVI, 2013). Segundo afirmam Helbing e Johansson (2011), os primeiros métodos de avaliação de pedestres baseavam-se em observações diretas, fotografias e vídeos em time-lapse. Esses estudos empíricos perduraram por mais de quatro décadas, tendo como um de seus principais objetivos desenvolver diretrizes de planejamento. Nesse ínterim, diferentes modelos de simulação foram propostos.

A dinâmica de pedestres pode ser dividida em situações normais e situações de pânico. Helbing et al. (2002a) afirmam que, embora os problemas característicos de cada uma dessas situações sejam caracterizados por diferentes fenômenos e investigados por diferentes comunidades científicas, eles podem ser tratados de maneira consistente por um mesmo modelo. Segundo Helbing e Johansson (2011), mesmo os modelos mais simples, que assumem respostas automáticas, têm potencial para bem reproduzir observações empíricas, sugerindo que interações simples podem levar a padrões inteligentes de movimento.

Alonso-Marroquin et al. (2012) também discorrem sobre o assunto e afirmam que, em função da dificuldade de realização de expe- rimentos com seres humanos, têm sido propostas simulações de partículas auto-dirigidas. Simulações estas que podem apresentar resultados contra intuitivos como, por exemplo, verificar benefícios na inserção de um obstáculo antes de uma saída. Benefícios que previnem ou reduzem o número de lesões sob condições de pânico em escapes, conforme demonstram os estudos de Helbing, Farkas e Vicsek (2000) e Escobar e De La Rosa (2003).

Este trabalho tem como delimitação simular e analisar cinco cenários diferentes para uma sala de aula com 32 alunos, tendo como referência inicial o estudo de Yang, Wu e Li (2011), que coletaram e analisaram gravações reais durante um terremoto no sudoeste da China, em 2008. Além disso, fundamenta-se no trabalho de Helbing et al. (2005), especialmente no que tange à configuração e distribuição do mobiliário interno de salas de aula. Tem-se como objetivo geral comparar os dados obtidos por Yang, Wu e Li (2011) com os dados simulados e como objetivos específicos: comparar o fluxo de evacuação (pedestres/segundo) para a mesma sala de aula com uma e com duas saídas e comparar o fluxo de evacuação para duas distribuições diferentes (simétrica e assimétrica) de mobiliário.

Diante de sua relevante influência no tempo total de evacuação em situações de emergência, o comportamento de pedestres tem sido o foco de muitas pesquisas no campo da física. Dificuldades técnicas e razões éticas, por outro lado, tornam raros os estudos sobre a fuga em pânico em situações reais de desastres, que Escobar e De La Rosa (2003) chamam de catástrofes naturais produzidas pelo homem, ou seja, catástrofes naturais pelo fato de não serem deliberadas. Daí a relevância das simulações computacionais.

O método de pesquisa adotado para o desenvolvimento deste estudo inclui a formulação de cinco diferentes cenários para a simulação de evacuação de uma sala de aula com 32 alunos em um software baseado em Forças 
Sociais. O trabalho está organizado em cinco partes, a saber: Introdução, Metodologia, onde constam os procedimentos metodológicos adotados e a fundamentação teórica, Resultados e Discussão e Conclusões.

\section{METODOLOGIA}

$\mathrm{Na}$ sequência serão apresentados a fundamentação teórica e o método de pesquisa adotado para o alcance dos objetivos supracitados.

\subsection{Dinâmica de pedestres e eva- cuação}

Considerando diferentes autores, Dias et al. (2013) afirmam que a dinâmica de pedestres em situações normais e de pânico apresenta-se por meio de diferentes fenômenos, tal qual descrito em Helbing et al. (2002a). Também conta com diferentes atributos psicológicos e emocionais. Os autores trazem ainda a questão da diminuição das habilidades cognitivas durante situações de pânico, corroborando com a pesquisa de Illera et al. (2010).

Frank e Dorso (2011) fazem uma análise histórica sobre desastres ocasionados por situações de pânico e afirmam que muito deles reforçaram o progresso na proteção à vida. Citam Grace Cheng et al. (2004) quando tratam da melhoria notável ocorrida na China, na última dinastia Qing (1644-1911 d.C.), quando se passou a exigir de forma estatutária que grandes edifícios possuíssem duas saídas de emergência. Fundamentados em Helbing, Farkas e Vicsek (2000), Frank e Dorso (2011) ponderam que essa solução depende fortemente do comportamento dos pedestres bem como da posição das saídas de emergência.

Yang, Wu e Li (2011) afirmam que o rastreamento de vídeos tem se apresentado como uma ferramenta valiosa para restringir-se modelos de simulação a partir da análise empírica de dados observacionais ou experimentais, tendo sido utilizado por diversos autores, dentre eles Helbing et al. (2003 e 2005) e Helbing, Johansson e Al-Abideen (2007). Afirmação esta que corrobora a importância do compartilhamento de informações e de lições aprendidas com situações de desastres já enfrentadas, conforme citado por Lima, Eyerkaufer e Gonçalves (2017). O desenvolvimento da economia e da sociedade como um todo, segundo Yang, Wu e Li (2011), tem proporcionado mais registros de vídeos com cenas de terremotos ou outros desastres, ainda que alguns deles apresentem baixa qualidade. Apesar de suas deficiências, a utilização desse material tem apresentado certa atratividade em função da natureza real dessas gravações, com grande importância para modelagens teóricas, conforme afirmam Helbing, Farkas e Vicsek (2000).

Segundo Moussaïd, Helbing e Theraulaz (2011), muitos modelos de comportamento de pedestres foram propostos a fim de explicar as leis subjacentes à dinâmica de multidões. Dentre eles, afirmam os autores, as abordagens que se baseiam na física são bastante comuns, a exemplo dos modelos de dinâmica fluida e de força social, ambos inspirados na mecânica newtoniana. Os modelos de força social descrevem o movimento de pedestres a partir de uma soma de forças atrativas e repulsivas, impulsivas e flutuantes, que refletem diversas influências externas bem como motivações internas.

\subsubsection{Modelo de Força Social}

A modelagem baseada em forças sociais de multidões de pedestres representa uma abordagem microscópica avançada que permite simular a dinâmica da movimentação de pedestres (SHUKLA, 2009). Esse tipo de modelagem, segundo o autor, tem sido utilizado de forma efetiva nas simulações de pedestres em situações normais e de pânico. Jiang et al. (2014) destacam que o modelo de força social 
é uma abordagem amplamente utilizada, capaz de modelar diversos comportamentos coletivos.

Para Helbing et al. (2005) o desejo de mover-se com determinada velocidade desejada é delineado por um termo de condução, enquanto a tendência de manter distância de outros pedestres, obstáculos, fronteiras e perigos reflete-se em forças repulsivas. Em situações normais, a velocidade desejada tem valor médio de $1,3 \mathrm{~m} / \mathrm{s}$, com desvio padrão aproximado de $0,3 \mathrm{~m} / \mathrm{s}$. Quando se faz necessário compensar atrasos, Helbing et al. (2005) apontam que a velocidade desejada é frequentemente aumentada com o passar do tempo. Para Helbing, Farkas e Vicsek (2002b), o fluxo simulado de um ambiente mostra-se bem coordenado e regular enquanto as velocidades desejadas são normais. Velocidades desejadas superiores a $1,5 \mathrm{~m} / \mathrm{s}$, no entanto, podem reduzir a eficiência da saída, o que é atribuído ao empurrão, que causa efeitos adicionais de fricção (HELBING et al., 2002a). Além disso, segundo os autores, para velocidades desejadas superiores a $5 \mathrm{~m} / \mathrm{s}$, as pessoas feridas tornam-se obstáculos imóveis para outras pessoas.

Uma vez calibradas com dados empíricos de fluxos de pedestres, as simulações computacionais produzem resultados realistas, mesmo quando se consideram novas geometrias e situações (HELBING et al., 2005). Assim sendo, o modelo de força social tem valor preditivo, permitindo a investigação de novos cenários, para os quais os experimentos se tornariam caros, complexos quanto a sua execução ou perigosos. Esse valor preditivo, segundo os autores, torna-se particularmente importante para o planejamento e otimização de rotas de fuga. Illera et al. (2010) atentam para o fato de que os potenciais dados de simulação, capazes de otimizar a segurança das pessoas em edifícios, devem ser incluídos no seu processo de planejamento. Da mesma forma, os resultados das diferentes pesquisas devem ser aplicados no projeto arquitetônico.

\subsubsection{Influência do Projeto das Áreas de Escape}

Pesquisas relacionados com a dinâmica de pedestres preveem que pequenas características arquitetônicas podem causar grandes efeitos sobre o fluxo de pedestres, especialmente nos casos de pânico (SHIWAKOTI; SARVI; BURD, 2014). Um pequeno e curto alargamento em um corredor, segundo Helbing, Farkas e Vicsek (2000), pode reduzir a velocidade média de escape de indivíduos em mais de 20\%. Corredores em ziguezague melhoram o fluxo de pedestres ao forçarem a alternância de direção e, consequentemente, da pressão exercida melos pedestres (HELBING et al., 2005). De maneira surpreendente, essas pesquisas também foram capazes de detectar que o fluxo de pedestres através de uma porta pode ser melhorado a partir da inserção de uma obstrução parcial à montante dela (HELBING et al., 2005).

Segundo Illera et al. (2010) o planejamento de evacuação não é um campo de pesquisa bem estabelecido na arquitetura. No entanto, o pânico e o estresse têm origem em espaços, que são o campo de ação natural dos arquitetos. Assim, os autores apontam para a necessidade de transpor os resultados de simulações de saída e tensão para a linguagem de arquitetos e planejadores. Acreditam que seja responsabilidade desses profissionais considerar os referidos resultados no processo de planejamento, tornando o projeto do edifício mais seguro. Shukla (2009) também defende que o desenho arquitetônico dos espaços exerce grande influência nos processos de evacuação e governa grande parte do fluxo de pedestres. E Jiang et al. (2014) afirmam que o projeto arquitetônico pode facilitar ou dificultar processos de fuga em situações de pânico.

Para Helbing et al. (2005), quando se traba- 
lha com uma otimização habilidosa do fluxo de pedestres, pode-se aumentar a eficiência e a segurança dessas instalações. Os autores destacam que esse aumento de eficiência pode vir acompanhando de custos compensadores e até redução de espaço. Desse modo, apresentam e discutem algumas soluções de projeto para entradas, saídas e rotas de fuga em estádios, teatros e salas de aula.

\subsubsection{Salas de Aula}

Embora as rotas de fuga sejam regulamentadas por lei, Helbing et al. (2005) afirmam que nem sempre é razoável que elas tenham largura constante. $\mathrm{O}$ número de pessoas que desejam sair aumenta a cada andar, pavimento ou fileira de assentos, à medida que as pessoas se aproximam da saída. Nesse sentido, o tempo para sair cresce não apenas em razão de longas distâncias de caminhada, mas também em razão dos tempos de espera em longas filas. $\mathrm{O}$ que pode resultar em impaciência na multidão, especialmente em situações onde o perigo é visível. Nesses casos, pode-se verificar o surgimento de comportamentos agressivos e suas possíveis consequências: fenômenos de entupimento, pessoas mortas e feridas. Assim sendo, larguras crescentes de rotas de fuga, semelhantes a rios, são consideradas um tipo de solução razoável, capaz de acelerar a evacuação de pessoas (HELBING et al., 2005).

Para Helbing et al. (2003), no caso específico de salas de aula, a distribuição de assentos de forma simétrica interfere em experimentos de evacuação, pois as pessoas utilizam especialmente a rota de fuga à direita. Assim, considerando o comportamento de orientação dos pedestres, as rotas de fuga devem estar do lado mais próximo à saída (Figura 1).
Figura 1. Tipos de distribuição de assentos em sala de aula de aula. Figure 1. Types of seating distribution in the classroom.

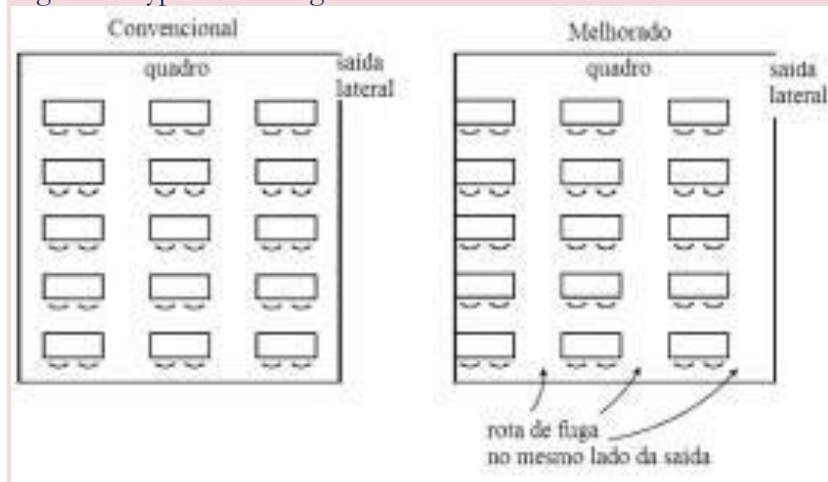

Fonte: Adaptado de Helbing et al. (2005).

Yang, Wu e Li (2011) coletaram e analisaram gravações reais realizadas durante um terremoto de magnitude 8 , no sudoeste da China, em 2008. Imagens publicadas pelos autores mostram o momento do terremoto em um espaço fechado, uma sala de aula com duas saídas, sendo que apenas a saída frontal aparece nas gravações. Yang, Wu e Li (2011) registraram o tempo que cada aluno levou para deixar a sala, de acordo com os lugares que ocupavam no momento do terremoto. Os alunos que saíram pela porta de trás aparecem claramente nas imagens, mas não foi possível quantificar o tempo de suas saídas devido ao enquadramento da câmera. Segundo os autores, todos os alunos apresentaram a tendência de escolher o caminho mais próximo.

Contrariando resultados de simulação, Yang, Wu e Li (2011) identificaram uma tendência de aceleração e não de dependência linear entre o tempo de evacuação e a ordem de saída das pessoas. Os autores não têm clareza sobre qual seria a causa da diferença entre os experimentos simulados e as observações da situação real. Para esclarecer qual subsistema é responsável por essa diferença, sugerem que novos estudos teóricos sejam desenvolvidos. 


\subsection{Método adotado para as simu- lações propostas}

A partir do estudo de Yang, Wu e Li (2011) propôs-se a simulação e análise de cinco diferentes cenários para uma sala de aula com 32 alunos. A primeira proposta (Cenário 1) busca replicar a sala de aula analisada por Yang, Wu e Li (2011), que contava com duas saídas. Segundo os autores, o processo de evacuação havia sido conduzido pelo professor. Em sua análise, Yang, Wu e Li (2011) puderam analisar os dados referentes aos 14 alunos que saíram pela porta da frente, já que a porta dos fundos da sala não estava no alcance da câmera de vídeo.

Os cinco cenários analisados neste estudo (Figura 2) têm como objetivo:

a) Cenário 1: reproduzir, para fins de comparação, a evacuação real de uma sala de aula com 32 alunos e duas saídas, uma frontal e outra posterior.

b) Cenário 2: analisar se há alterações no tempo de evacuação observado no Cenário 1 no caso de haver apenas uma saída na sala de aula (frontal).

c) Cenário 3: verificar se o posicionamento do mobiliário da sala de aula influi no tempo de evacuação do Cenário 1.

d) Cenário 4: verificar se o posicionamento do mobiliário da sala de aula influi no tempo de evacuação do Cenário 1 no caso de haver apenas uma saída (frontal).

e) Cenário 5: verificar se há alteração no tempo de evacuação do Cenário 1 quando os alunos podem optar por qualquer uma das duas saídas.
Figura 2. Cenários simulados.

Figure 2. Simulatedscenarios.

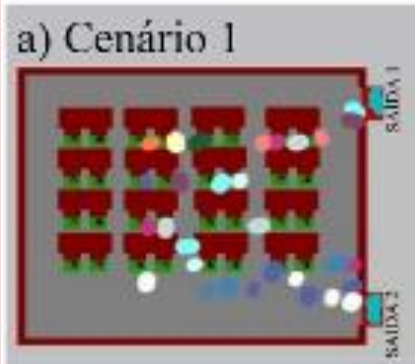

b) Cenário 2

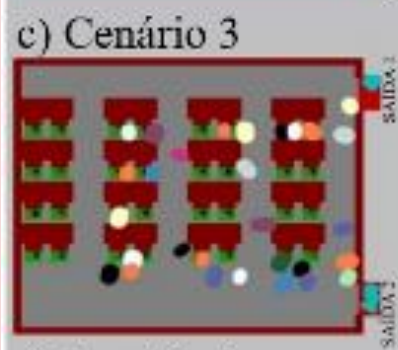

d) Cenário 4
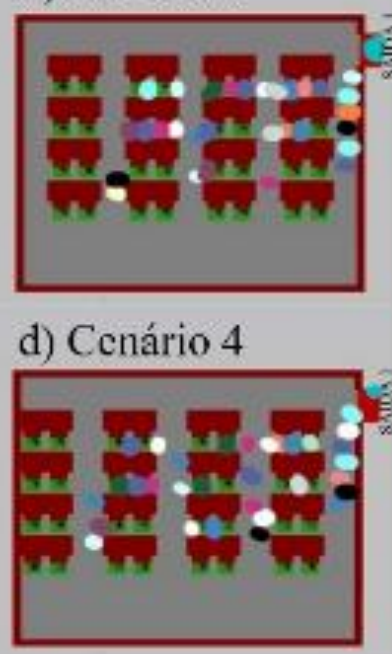

c) Cenário 5

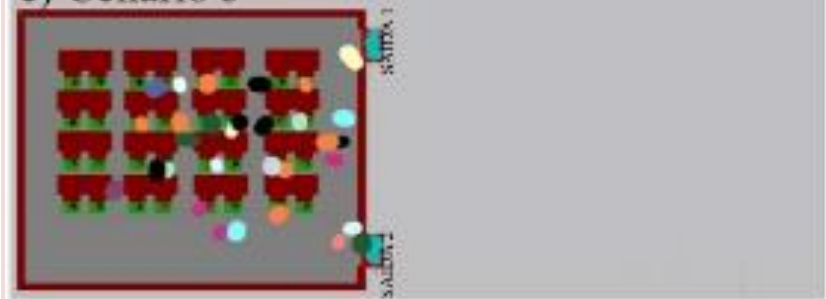

Fonte: Imagem obtida por captura de tela do software PTV Viswalk após 1,4 s do início da simulação.

Importante destacar que as dimensões da sala de aula foram inferidas a partir da dimensão padrão de uma carteira escolar dupla (NEUFERT, 1976), para a qual adotou-se 125 $\mathrm{cm}$ de comprimento. Por não constar nos desenhos e imagens do estudo de Yang, Wu e Li (2011), a parte posterior da sala de aula foi arbitrada, incluindo a posição e a largura da saída 2. Portanto, adotou-se para esta saída a mesma largura da saída 1, posicionando-a no ponto em que o desenho apresentado pelos autores indica a ausência de informação sobre as dimensões exatas da sala de aula (linha tracejada), distante $4,80 \mathrm{~m}$ da primeira saída, aproximadamente. Num primeiro momento, procurou-se simular os cenários com a parte posterior totalmente aberta, tendo como foco exclusivo os alunos que evacuavam pela saída 1. No entanto, essa estratégia mostrou resultados bastante divergentes da situação real, 
indicando que o percurso dos 18 alunos até a saída 2 interferiu no tempo de evacuação dos 14 alunos que abandonaram a sala de aula pela saída 1.

O posicionamento das carteiras nos Cenários 1, 2 e 5 obedece à disposição indicada por Yang, Wu e Li (2011). Já o posicionamento das carteiras nos Cenários 3 e 4 busca avaliar a eficácia da assimetria do mobiliário, indicada por Helbing et al. (2005). Os Cenários 2 e 4 buscam avaliar se há interferência no tempo de evacuação da sala de aula no caso de haver apenas uma saída. Reforça-se que, no Cenário 5 , a configuração das rotas de evacuação permite aos alunos destinarem-se a qualquer uma das duas saídas, segundo a sua escolha. Já nos Cenários 1 e 3, buscou-se reproduzir a orientação do professor no momento da evacuação da sala de aula e/ou o treinamento prévio dos alunos para situações emergenciais. Para tanto, determinou-se a configuração da rota de evacuação de cada um dos alunos, destinando-os às saídas 1 ou 2 , obrigatoriamente.

As simulações foram realizadas no software PTV Viswalk, que foi desenvolvido pelo grupo alemão PTV Planung Transport Verkehr AG e é capaz de simular e modelar o comportamento de pedestres. Baseada no modelo de Forças Sociais, a ferramenta se utiliza de variáveis do comportamento humano como evitar colisões com outros pedestres e obstáculos. Cada cenário foi simulado 20 vezes para uma mesma velocidade desejada, igual a $\mathrm{Vd}=1,53$ $\mathrm{m} / \mathrm{s}$.

\section{RESULTADOS E DISCUSSÃO}

Observa-se, na Figura 3, os cenários 1, 2 e 5 após 1,5 s do início da evacuação. Ressaltase que, no Cenário 1, os alunos foram conduzidos para as saídas frontal (14 alunos) ou posterior (18 alunos), conforme apontado por Yang, Wu e Li (2011). A Figura 4 apresenta o tempo de evacuação (em segundos) e a posição dos alunos que abandonaram a sala de aula pela saída frontal nos cenários real e simulado. Pode-se observar que a simulação apresentou, aproximadamente, um fluxo de evacuação 61\% maior que o cenário real. O que pode ser atribuído à velocidade de caminhada ou ao tempo de reação dos alunos após o terremoto, por exemplo. Especialmente pelo fato dos alunos mais próximos à saída terem apresentado tempos de evacuação relativamente altos no cenário real. As carteiras hachuradas estavam ocupadas pelos alunos que utilizaram a saída 2 .

Figura 3. Instantâneos da evacuação no Cenário 1 (esquerda), no Cenário 2 (direita) e no Cenário 5 (abaixo).

Figure 3. Evacuation snapshots in Scenario 1 (left), Scenario 2 (right), and Scenario 5 (below).

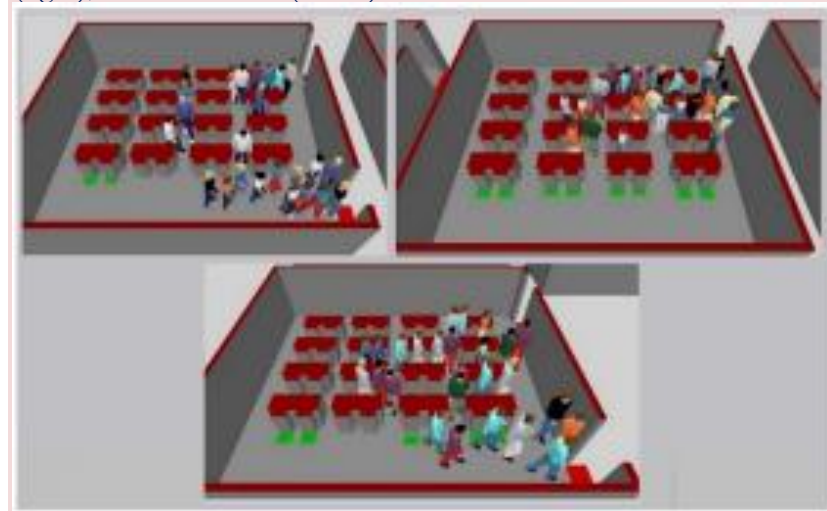

Fonte: Imagem obtida por captura de tela do software PTV Viswalk após $1,5 \mathrm{~s}$ do início da simulação. 
Figura 4. Tempo de evacuação pela saída 1 no cenário real (acima) e no Cenário 1, simulado (abaixo).

Figure 4. Evacuation time for output 1 in the real scenario (above) and Scenario 1, simulated (below).

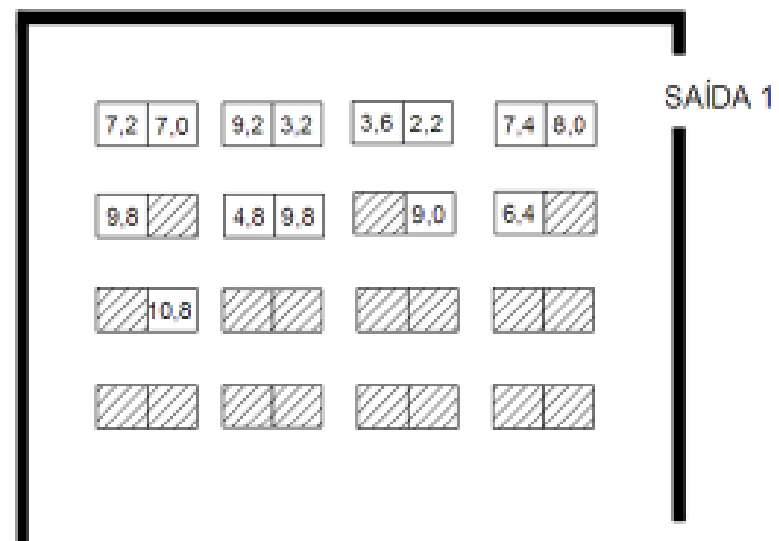

SAIDA 2

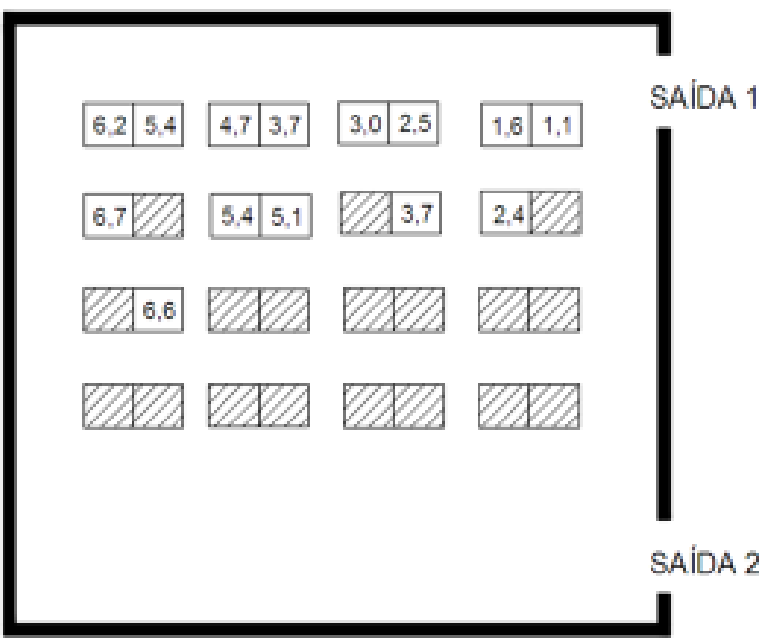

Fonte: Autoria própria.

O Gráfico 1 relaciona a ordem e o tempo de saída dos 14 alunos que abandonaram a sala de aula pela saída 1 (frontal). Em azul vêse o resultado da simulação e em alaranjado o resultado real apresentado por Yang, Wu e Li (2011). A comparação de ambos os resultados reforça a suspeita dos autores de que o comportamento das pessoas durante a evacuação parece ter natureza diferente das simulações. Isso porque o tempo e a ordem de abandono parecem linearmente dependentes na simulação, mas exibem tendência de aceleração na evacuação real.
Gráfico 1. Comparação entre cenário real e simulado.

Graph 1. Comparison between real and simulated scenario.

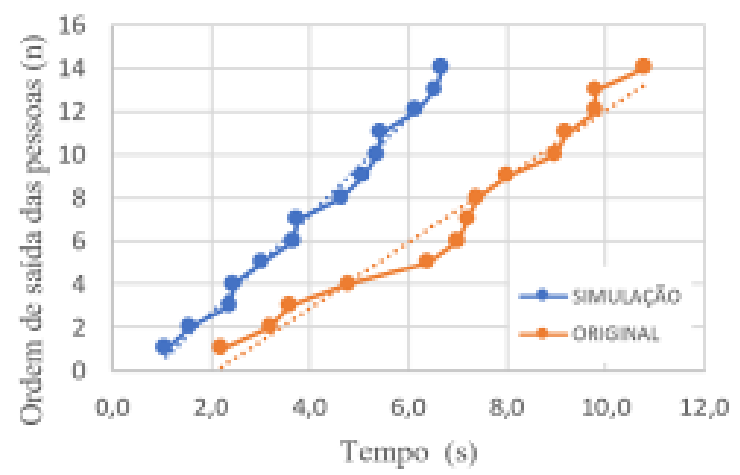

Fonte: Autoria própria.

O Gráfico 2 apresenta o fluxo de evacuação de pedestres calculado para cada um dos cinco cenários simulados. Tratam-se de valores médios obtidos a partir de 20 simulações para cada cenário, dos quais considerou-se apenas o tempo final de evacuação, independentemente da saída (frontal ou posterior) bem como o número total de alunos (32 alunos).

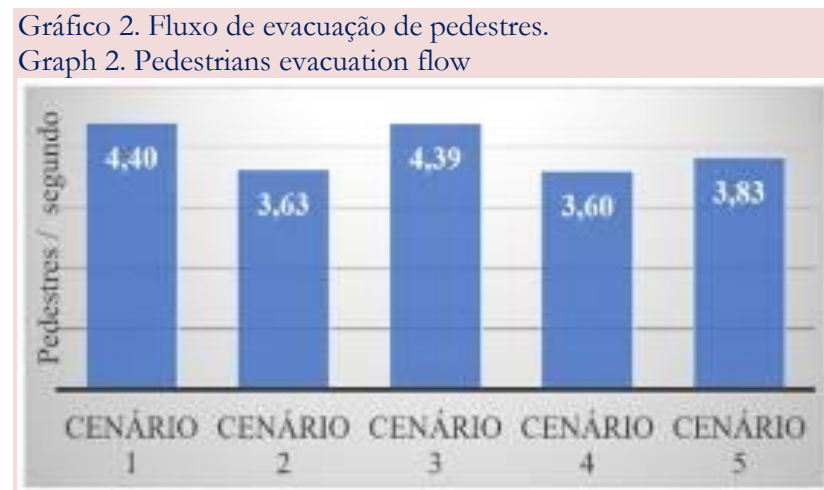

Fonte: Autoria própria.

Os resultados das simulações indicam que o Cenário 1 apresenta o melhor desempenho no que diz respeito ao fluxo de evacuação de pedestres, com uma taxa de 4,40 pedestres / segundo. Eles confirmam, portanto, a afirmativa de que a existência de duas saídas, ao invés de uma, aumenta o fluxo de evacuação de pedestres. O que ficou evidenciado nos resultados dos Cenários 2 e 4, para os quais obteve-se, respectivamente, redução de $17,56 \%$ e 
18,07\% no fluxo de pedestres em relação ao Cenário 1. É possível inferir ainda que, no estudo proposto, a assimetria do mobiliário da sala de aula não interferiu no fluxo de evacuação, pois praticamente não há diferença entre o fluxo de pedestres apresentado pelos Cenários 1 e 3 (com duas saídas cada) e tampouco entre os cenários 2 e 4 (com 1 saída cada). É importante destacar que, embora a largura média dos corredores entre as carteiras tenha aumentado cerca de 100\% com o novo layout, para que todos os corredores apresentassem a mesma dimensão, a largura do corredor junto às saídas sofreu decréscimo de, aproximadamente, $21 \%$.

Por fim, comparando-se o resultado do Cenário 5, em que os alunos estavam livres para escolher a sua rota de fuga (saída frontal ou posterior), com o resultado do Cenário 1, em que 14 alunos foram conduzidos para a saída frontal e 18 para a saída posterior, vê-se que o Cenário 5 sofreu uma queda de 12,98\% no fluxo de evacuação. Considerando-se que o Cenário 1 buscou replicar a situação real, em que a evacuação da sala de aula durante a emergência foi conduzida pelo professor, o resultado demonstra a importância do treinamento prévio dos alunos, professores e funcionários para situações emergenciais. Treinamento este que representou, nas simulações realizadas, uma eficácia comparável àquela representada pela existência duas ao invés de uma única saída e que, portanto, não pode ser negligenciado.

\section{CONCLUSÕES}

Os resultados alcançados com esta pesquisa reforçam o valor preditivo das ferramentas de simulação computacional, especialmente aquelas baseadas no modelo de Força Social. Característica que está fortemente relacionada à busca de novas soluções projetuais que visem a segurança das pessoas e a preservação da vida em situações de emergência que exi- gem a evacuação de pedestres. As comparações entre os cinco cenários simulados destacam a efetividade da adoção de duas saídas para as configurações de salas de aulas testadas e não demonstram diferenças expressivas entre as distribuições simétricas e assimétricas do mobiliário avaliado. No entanto, sugere-se, para trabalhos futuros, que sejam testadas novas distribuições, privilegiando-se o corredor mais próximo à(s) saída(s) ao conferir-lhe maior largura que aos demais corredores. Por fim, os resultados alertam para a necessidade de treinamento de alunos, professores e funcionários para situações de emergências, o que pode gerar aumentos consideráveis nos fluxos de evacuações e, portanto, a mitigação de riscos de perdas de vidas humanas.

\section{REFERÊNCIAS}

ALONSO-MARROQUIN, F. et al. Bottlenecks in granular flow: When does an obstacle increase the flow rate in an hourglass? Physical Review E, v. 85, n. 2, p. 020301(1-4), 2012. https://doi.org/10.1103/PhysRevE.85.020301 GRACE CHENG, W. Y. et al. A view on the means of fire prevention of ancient Chinese buildings-from religious belief to practice. Structural Survey, v. 22, n. 4, p. 201-209, 2004. https://doi.org/10.1108/02630800410563741

DIAS, C. et al. Investigating collective escape behaviours in complex situations. Safety Science, v. 60, p. 87-94, 2013. https://doi.org/10. 1016/i.ssci.2013.07.005

ESCOBAR, R.; DE LA ROSA, A. Architectural design for the survival optimization of panic King fleeing victims. In: EuropeanConferenceon Artificial Life. Springer, Berlin, Heidelberg, 2003. p. 97-106. https://doi.org/ 10.1007/978-3-540-39432-7_11

FRANK, G. A.; DORSO, C. O. Room evacuation in the presence of an obstacle. Physica A: Statistical Mechanics and its Applications, v. 390, n. 11, p. 2135-2145, 2011. https://doi.org/10.1016/i.physa.2011.01.015

HELBING, Dirk et al. Self-organized pedes- 
trian crowd dynamics: Experiments, simulations, and design solutions. Transportation Science, v. 39, n. 1, p. 1-24, 2005. https://doi. org $/ 10.1287 / \operatorname{trsc} .1040 .0108$

HELBING, Dirk et al. Simulation of pedestrian crowds in normal and evacuation situations. Pedestrian and Evacuation Dynamics, v. 21, n. 2, p. 21-58, 2002a.

HELBING, D.; FARKAS, I.; VICSEK T. Crowd Disasters and Simulation of Panic Situations. The Science of Disasters. Springer, Berlin, Heidelberg, p. 330-350, 2002b. https://doi.org/10.1007/978-3-642-56257$\underline{0} 11$

HELBING, D.; FARKAS, I.; VICSEK, T. Simulating Dynamical Features of Escape Panic. Nature, v. 407, n. 6803, p. 487, 2000. https://doi.org/10.1038/35035023

HELBING, D. et al. Lattice gas simulation of experimentally studied evacuation dynamics. Physical Review E, v. 67, n. 6, p. 067101 , 2003. https://doi.org/10.1103/PhysRevE.67.067101 HELBING, D.; JOHANSSON, A. Pedestrian, Crowd and Evacuation Dynamics. In: MEYERS, R. (eds) Extreme Environmental Events. Springer, New York, NY, 2011. https://doi.org/10.1007/978-1-4419-7695-6 37 HELBING, D.; JOHANSSON, A.; ALABIDEEN, H. Z. Dynamics of crowd disasters: An empirical study. Physical Review E, v. 75, n. 4, p. 046109, 2007. https://doi.org/10. 1103/PhysRevE.75.046109

ILLERA, C. et al. NO_PANIC. Escape and panic in buildings-architectural basic research in the context of security and safety research. In: KLINGSCH, W. et al. (Eds.). Pedestrian and Evacuation, Dynamics 2008. SpringerVerlag Berlin, Heidelberg, p. 733-742, 2010. https://doi.org/10.1007/978-3-642-04504-2 71

JIANG, Li et al. Obstacle optimization for panic flow-reducing the tangential momentum increases the escape speed. PloS One, v. 9, n. 12, p. e115463, 2014. https://doi.org/10.1371/ journal.pone. 0115463

LIMA, F. S.; EYERKAUFER, M. L.; GONÇALVES, M. B. 2017. Gestão de desastres.
In: LEIRAS, A. et al. [Orgs]. Logística Humanitária. 1 ed. Rio de Janeiro: Elsevier, 2017, p. 57-77.

MOUSSAÏD, M.; HELBING, D.; THERAULAZ, G. How simple rules determine pedestrian behavior and crowd disasters. Proceedings of the National Academy of Sciences, v. 108, n. 17, p. 6884-6888, 2011. https://doi.org/10.1073/pnas.1016507108

NEUFERT, E. Arte de Projetar em Arquitetura: Princípios, normas e prescrições sobre construção, instalações, distribuição e programa de necessidades, dimensões de edifícios, locais e utensílios. 5. ed. Trad. $21^{\mathrm{a}}$ ed. Alemã, São Paulo: Gili do Brasil, 1976.

SHIWAKOTI, N.; SARVI, M. Enhancing the panic escape of crowd through architectural design. Transportation Research part C: Emerging Technologies, v. 37, p. 260-267, 2013. https://doi.org/10.1016/j.trc.2v013.04.009 SHIWAKOTI, N.; SARVI, M.; BURD, M. Using non-human biological entities to understand pedestrian crowd behaviour under emergency conditions. Safety Science, v. 66, p. 1-8, 2014. https://doi.org/10.1016/j.ssci.2014.01. $\underline{010}$

SHUKLA, P. K. Genetically optimized architectural designs for control of pedestrian crowds. In: Australian Conference on Artificial Life. Springer, Berlin, Heidelberg, 2009. p. 22-31. https://doi.org/10.1007/978-3$\underline{642-10427-5 \quad 3}$

YANG, X.; WU, Z.; LI, Y. Difference between real-life escape panic and mimic exercises in simulated situation with implications to the statistical physics models of emergency evacuation: The 2008 Wenchuan earthquake. Physica A: Statistical Mechanics and its Applications, v. 390, n. 12, p. 2375-2380, 2011. https://doi.org/10.1016/i.physa.2010.10.019

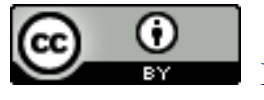
access article distributed under the terms of the Creative Commons Attribution License, which permits unrestricted use, distribution, and reproduction in any medium, provided 
the original work is properly cited.

Artigo recebido em 12 de fevereiro de 2019.

Avaliado em 06 de setembro de 2019.

Aceito em 06 de setembro de 2019.

Publicado em 02 de outubro de 2019.

\section{Como citar este artigo (ABNT):}

NAPPI, Manuela Marques Lalane; MOSER, Ivana Righetto; SOUZA, João Carlos. Avaliação do tempo de evacuação de salas de aula com diferentes soluções de projeto. Estação Científica (UNIFAP), Macapá, v. 9, n. 1, p. 39-49, jan./mar. 2019. 